\title{
Quality of Life in Australian Women with Female Pattern Hair Loss
}

\author{
Sebastiana Biondo and Rodney Sinclair ${ }^{*}$
}

Department of Medicine, University of Melbourne, Melbourne, Australia; Department of Dermatology, St. Vincent's Hospital and the Skin and Cancer Foundation, Victoria, Australia

\begin{abstract}
Background: Female pattern hair loss (FPHL) produces chronic progressive hair loss in women. The morbidity is predominately psychological. Empirical research investigating the psychological aspects of FPHL and the impact of initiation of treatment in Australian women is absent.

Objective: To examine the impact of FPHL on the quality of life (QOL) and the impact of initiation of treatment on QOL.

Method: 77 women with biopsy-proven FPHL receiving treatment with oral antiandrogens, 53 women on a waiting list to receive treatment for FPHL and 82 women not affected by hair loss completed the Women's AGA Quality of Life Questionnaire (WAA-QOL).

Results: AGA affected women reported poorer health-related QOL than non-affected women. There was no change in QOL over a two-month period following assessment and initiation of treatment. The WAA-QOL was found to have excellent internal consistency (Cronbach's alpha $=.97)$ and test-retest reliability (correlation coefficient $=.92)$.

Conclusion: The findings confirm the detrimental impact of FPHL on the QOL and the lack of impact of medical consultation and initiation of treatment on QOL at 2 months. This has implications for the treatment of psychological difficulties associated with FPHL and suggests a possible role for adjuvant psychological intervention.
\end{abstract}

Keywords: Androgenetic alopecia, psychological impact, health-related QOL.

\section{INTRODUCTION}

For many the appearance of one's hair is central to feelings of attractiveness and self-esteem [1].

The most common type of hair loss in men [2] is androgenetic alopecia (AGA) and in women is female pattern hair loss (FPHL) [3-5]. FPHL typically develops as diffuse thinning over the crown [6]. A population survey carried out in 2001 indicated that the age adjusted prevalence of FPHL among Australian women aged 20 and over is $32.2 \%$ (95\% CI, $28.8 \%-35.6 \%$ ) of whom $10.5 \%$ (95\% CI, $8.2 \%-12.7 \%$ ) or approximately 800,000 Australian women have moderate to severe FPHL (Fig. 1) [7]. The onset of FPHL can be distressing and confusing for sufferers, as FPHL is not well understood or anticipated [1].

A number of studies have addressed the psychosocial difficulties men experience as a result of their hair loss [817]. Investigations of the impact of FPHL indicate that patterned hair loss is more distressing for women [18-20].

Medical treatments, such as antiandrogen therapy and topical preparations may simply arrest progression of FPHL or in a minority stimulate partial regrowth of hair [21-23], however, the response is slow.

Most research has examined the psychosocial impact of AGA using generic health-related quality of life (HRQOL)

*Address correspondence to this author at the Department of Dermatology, St. Vincent's Hospital, 41 Victoria Parade, Fitzroy, VIC 3065, Australia; Tel: +61392883298; Fax: +61392883544; E-mail: rod.sinclair@svhm.org.au scales that are not specific to the condition [13]. It is often the case that generic measures of HRQOL are not sensitive to quality of life issues associated with the specific medical conditions [13]. Condition-specific measures are required to uncover the concerns of patients, the areas of functioning specifically affected by the condition and to track changes post-treatment $[24,25]$. To date, only one measure exists that assesses HRQOL specific to women with hair loss - the Women's AGA Quality of Life Questionnaire (WAA-QOL) [26].

The present study examines the impact of FPHL and its treatment on the quality of life of affected women as measured using the WAA-QOL [26]. In addition we examine the impact of consultation and initiation of treatment on women with FPHL using the WAA-QOL.

\section{METHOD}

\section{Participants}

\section{Recruitment}

Participants comprised of three groups: treatment; waiting list; and normal control.

Participants in the treatment group were drawn from a population of women with biopsy-proven AGA who were undergoing treatment for the condition. A terminal to vellus ratio of less than or equal to $4: 1$ on horizontally sectioned scalp biopsy taken from the mid-frontal scalp is considered diagnostic for AGA. Treatment included oral anti-androgens (either spironolactone or cyproterone acetate) alone or combined with topical minoxidil 5\% lotion. 


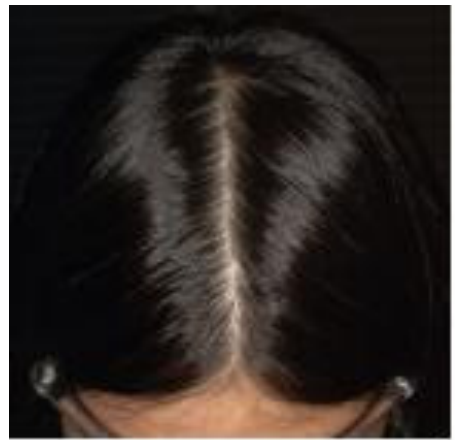

1

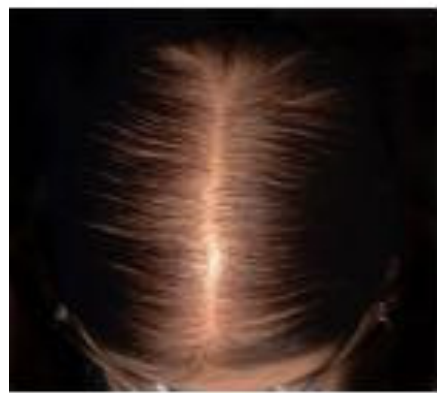

2

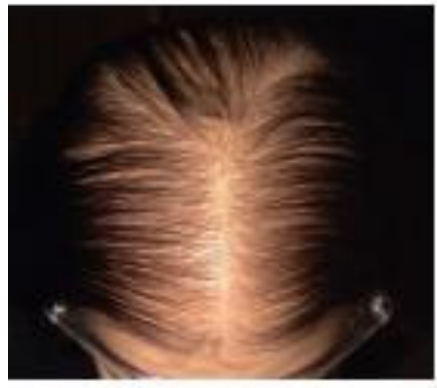

3

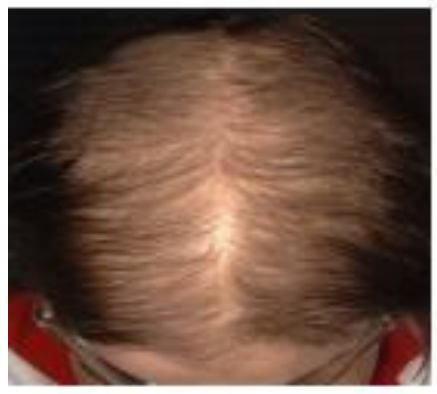

4

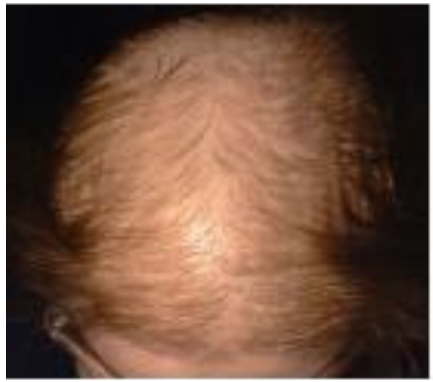

5

Fig. (1). Female pattern hair loss clinical grading scale. Stage 1 is considered normal, stage 2 indicates mild FPHL. Stages 3, 4 and 5 indicate moderate-to-severe hair loss.
Participants in the waiting list group were waiting for an appointment to be seen by a dermatologist in a hair loss clinic. They each had a one hour appointment during which a comprehensive history was taken, the scalp was examined, a battery of blood tests was performed and three $4 \mathrm{~mm}$ punch biopsies were taken from the scalp for histological assessment. A detailed explanation of the normal hair cycle and pathogenesis of androgenetic alopecia was given to each patient. An opportunity to ask questions and have specific concerns addressed was provided. In addition each patient was given a printed leaflet to reinforce the information provided.

On review at 2 weeks all patients were given a definite diagnosis based on the histology results; their blood test results; and the possible benefits of the proposed medications and any potential side-effects were discussed in detail and additional leaflets were provided regarding the use of that medication to treat androgenetic alopecia and the likely time course of effect. Women were told that the natural history of FPHL is for slowly progressive hair loss at a rate of about $5 \%$ per year and that the primary goal of therapy was to arrest this progression. Patients were instructed that while hair regrowth can recur, it is slow, never complete and unpredictable. Treatment consisted of an oral anti-androgen (either spironolactone or cyproterone acetate) alone or in combination with topical minoxidil lotion.

Control group participants were recruited from among patrons of hairdressing salons in the Melbourne suburbs of Malvern, Mt Waverley and Oakleigh who responded to recruitment posters.

\section{Materials}

Participants were issued with a questionnaire that consisted of an explanatory statement, demographic items including age, marital status, and country of birth and the WAA-QOL questionnaire.

\section{WAA-QOL}

The WAA-QOL is a 16 item self-report measure of HRQOL in women with AGA. Respondents were required to indicate the degree to which the experiences described in the items have happened to them in the past week. Responses were recorded on a seven point Likert scale ranging from $0=$ "not at all" to $6=$ "extremely". Responses are then totalled, with possible scores ranging from 0 (high HRQOL) to 96 (low HRQOL). While, in its original form, items in the WAA-QOL refer specifically to hair loss, in the present study the words "hair loss" were replaced by "problems with your hair" (Table 1). This was done so that the WAA-QOL would be applicable to both AGA and non-AGA affected populations.

The authors of the WAA-QOL have reported initial psychometric data, based on a sample of 137 postmenopausal women with thinning hair. The questionnaire demonstrated excellent test-retest reliability (.89) and high internal consistency (.98).

\section{Procedure}

Participants in the treatment and waiting list populations were mailed the questionnaire. Potential participants in the 
Table 1. The Women's AGA Quality of Life Questionnaire (WAA-QOL)

Note. The words "hair loss" were replaced by "problems with your hair" so that it would be applicable to both AGA and non-AGA Australian populations. Please read the items below and circle the response that best matches your feelings.

For each question choose from the following alternatives: $0=$ not at all $1=$ a little bit $2=$ somewhat $3=$ a good bit $4=$ quite a bit $5=$ very much $6=$ extremely

1. In the past week, how self-conscious have you been about people looking at your hair?

2. In the past week, how jealous/envious have you been about other people who have lots of hair?

3. In the past week, how much have problems with your hair negatively affected your self-confidence?

4. In the past week, how unattractive have you felt because of the problems with your hair?

5. In the past week, how much was socialising with people you didn't know uncomfortable for you because of the problems with your hair?

6. In the past week, how much was interacting with the opposite sex (or same sex) uncomfortable for you because of the problems with your hair?

7. In the past week, how much has problems with your hair negatively affected your satisfaction with the appearance of your hair?

8. In the past week, has problems with your hair negatively affected the way you like to style your hair?

9. In the past week, how powerless (lack of control) have you felt to do anything about the problems with your hair?

10. In the past week, how embarrassed have you felt because of the appearance of your hair?

11. In the past week, how frustrated have you felt because of the problems with your hair?

12. In the past week, how concerned have you been about your hair parting and showing your scalp?

13. In the past week, how concerned have you been that the problems with your hair will continue?

14. In the past week, how much time have you spent making your hair look fuller/thicker because of the problems with your hair?

15. In the past week, how annoyed have you been at having to spend time fixing your hair because of the problems with your hair?

16. In the past week, how much time have you spent checking your hair in the mirror because of the problems with your hair?

control population were given the same questionnaire by their hairdresser on request and in response to recruitment posters.

\section{RESULTS}

\section{Stage 1 Participation}

A total of 550 questionnaires were distributed. Of these, 222 were returned; 77 from the treatment population, 57 from the waiting list population and 88 from the control population, resulting in a $40.7 \%$ response rate. Of the 222 respondents, four respondents from the waiting list sample described symptoms of alopecia areata, a condition unrelated to FPHL, and were excluded from the study. Six respondents from the control group were excluded as they described symptoms of hair loss. This reduced the number of participants to 212 women; 77 in the treatment group, 53 in the waiting list group, and 82 in the control group. The mean age of participants in the treatment group was 42.29 years $(S D=12.34)$, the mean age in the waiting list group was 44.92 years $(S D=15.81)$ and the mean age in the control group was 38.38 years $(S D=12.34)$. Of the final sample, $45.3 \%$ had completed tertiary education, $47.9 \%$ were employed as professionals, $72.6 \%$ were born in Australia or New Zealand and $49.6 \%$ were married.

\section{Stage 2 Participation}

Participants were asked to complete the questionnaire administered in Stage 1 again after a two-month period. Of the original sample, 127 participated in Stage 2; 47 from the treatment group, 30 from the waiting list group, and 50 from the control group, resulting in a $59.9 \%$ response rate.

\section{Analysis of Questionaire Results}

Descriptive statistics for age and WAA-QOL scores at time 1 and time 2, for the three groups (treatment, waiting list and control groups) are shown in Table 2.

Median and semi-interquartile range (SIQR) scores were calculated for each of the WAA-QOL items to assess which were perceived as most distressing to women with AGA and are presented in Table 3 .

Table 2. Means and Standard Deviations for Age and WAA-QOL Scores at Time 1 and Time 2, for the Three Groups

\begin{tabular}{|c|c|c|c|c|c|c|c|c|c|}
\hline & \multicolumn{3}{|c|}{ Treatment } & \multicolumn{3}{c|}{ Waiting List } & \multicolumn{3}{c|}{ Control } \\
\cline { 2 - 10 } & N & M & SD & N & M & SD & N & M & SD \\
\hline \hline Age time 1 & 77 & 45.29 & 12.34 & 53 & 44.92 & 15.81 & 82 & 38.38 & 0.92 \\
\hline WWA-QOL time 1 & 77 & 2.87 & 1.72 & 53 & 3.08 & 1.53 & 82 & 0.82 & 0.92 \\
\hline WWA-QOL time 2 & 47 & 2.94 & 1.59 & 30 & 3.37 & 1.58 & 50 & 0.26 & 0.43 \\
\hline
\end{tabular}


Table 3. Median and SIQR Scores for the Sixteen Items on the WAA-QOL for FPHL Affected Women

\begin{tabular}{|l|c|c|c|c|c|}
\hline & Median & SIQR & & Median & SIQR \\
\hline \hline Q1 & 2.00 & 1.50 & Q9 & 3.00 & 2.50 \\
\hline Q2 & 3.00 & 2.00 & Q10 & 2.00 & 1.50 \\
\hline Q3 & 2.00 & 1.50 & Q11 & 3.50 & 2.00 \\
\hline Q4 & 3.00 & 1.50 & Q12 & 4.00 & 2.00 \\
\hline Q5 & 1.00 & 1.50 & Q13 & 5.00 & 2.00 \\
\hline Q6 & 1.00 & 1.50 & Q14 & 3.00 & 2.00 \\
\hline Q7 & 4.00 & 1.50 & Q15 & 3.00 & 2.00 \\
\hline Q8 & 4.00 & 1.50 & Q16 & 3.00 & 2.00 \\
\hline
\end{tabular}

The results presented in Table $\mathbf{3}$ indicate that median scores were very high for Q.13 (concern about hair loss continuing), Q.7 (dissatisfaction with their appearance), Q.8 (inability to style their hair) and Q.12 (concern about hair parting and showing scalp). Emotional aspects were also high, including Q.2 (jealousy), 4 (feeling unattractive), 9 (feeling powerless to stop their hair growth) and 11 (frustrated).

To test whether there was a significant difference in WAA-QOL total scores between those women with FPHL and those women without FPHL; an independent group's $t$ test was performed. Treatment and waiting list groups were combined to form the FPHL group. It was found that WAAQOL scores for the FPHL group were significantly higher than those of the control group $(t(207)=12.10, \mathrm{p}<.001)$.

A mixed factorial ANOVA $(2 \times 3$, time $\mathrm{x}$ group $)$ involving both between- and within-subjects factors was conducted to test whether the WAA-QOL scores changed over the two-month period for the treatment, waiting list and control groups. The results for the mixed factorial ANOVA indicated that there was no significant interaction between time and group $(F(2,124)=0.74, p=.48)$. The main effect for time was not significant; the WAA-QOL scores did not change significantly from the initial measurement to two months later $(F(1,124)=3.12, p=.08)$. A significant main effect for group membership was found $(F(2,124)=78.37, p$ $<.001)$.

To assess where the significant effect for group lies, post hoc analyses were performed. Tukey's post-hoc analysis revealed that there was a significant difference between the control and the treatment group and between the control and waiting list group (for both, $p<.001$ ). There was no significant difference between the treatment and waiting list groups $(p=.31)$.

\section{DISCUSSION}

This study explores the psychological impact of FPHL in Australian women. The study revealed that Australian women with AGA experience poorer HRQOL than women without hair loss. This finding is consistent with previous findings that female AGA can be psychologically debilitating and may reflect the assumption that a women's self-esteem is closely tied to their appearance [18-20, 26]. The AGA affected women involved in the present study, however, were all actively receiving or seeking treatment.

It can be argued that due to the wording of the questionnaire i.e. the use of "problems with your hair" there is a bias in the questionnaire with the result that women in the control group with no hair loss will automatically rate low unless they perceive that they have a hair problem. However this phrase was chosen to replace "hair loss" in the original questionnaire as this helps to differentiate effects on quality of life of physiological problems with hair such as white hairs, frizzy, brittle or unmanageable hairs as opposed to the pathological problem or hair loss.

Over a two-month period, there was no improvement in HRQOL for those women on treatment. This is not surprising given that the anticipated response to any therapy will be slow [22]. The impact of treatment upon quality of life may be better understood in a longitudinal study. Similarly, there was no improvement in HRQOL in the waiting list control group over the two-month period.

The study allowed for the identification of the aspects of hair problems that women perceive as most distressing or as having the greatest impact on day to day life. The item perceived as most important to women with AGA referred to concern about hair loss continuing. Also of importance were dissatisfaction with appearance, inability to style hair and concern about hair parting and scalp showing. Emotional aspects associated with hair loss in women were also of concern to patients. These included feelings of jealousy/envy about other people having thick hair, feeling unattractive, feeling powerless to stop hair loss and frustration. Other concerns, such as feeling uncomfortable socialising with people and uncomfortable interacting with the opposite sex (or same sex), were less important to AGA affected women. These findings are consistent with research that assessed the relevance and importance of 190 thoughts and feelings previously identified as being of relevance to females with AGA [27].

Specific limiting features include sample characteristics and time frame. Sample characteristics that should be acknowledged include the fact that all AGA affected women were 'treatment seekers' and that the length of time in treatment varied across members of the treatment group. Women seeking medical treatment may be motivated, in large part, by the substantial distress and psychological impairment that they experience in relation to their condition. Furthermore, it may be that changes in HRQOL may be expected at different stages during treatment (e.g. at 6 months, 12 months, etc.). It was unknown at what stage in treatment members of the treatment group were at the time of the study.

This study indicates that consultation and initiation of treatment does not on its own improve QOL for women with FPHL. Further research is required to establish the long term benefits of oral antiandrogen therapy on QOL and to investigate the possible role for adjuvant psychological intervention in the treatment of hair loss.

Illness perceptions have been demonstrated to have an independent effect on QoL in a number of chronic diseases 
and it has been suggested that modification of these perceptions, especially in difficult to treat diseases may be an effective strategy to improving well-being. The impact of female pattern hair loss is influenced by both disease related factors such as the extent and visibility of hair loss, as well as psychological factors such as beliefs about hair loss in women, and behavioural and coping factors [28]. Cartwright et al. [29] demonstrated that in women with alopecia areata perceptions of the nature and severity of alopecia account for $35 \%$ of the total variance in DLQI scores between women with and without alopecia. The most important illness perceptions in their cohort were illness identity (associating more symptoms with their condition), belief in the serious consequences of alopecia, and a strong emotional response to the condition. Pending improved therapies, this approach also merits consideration in the management of women affected by FPHL.

\section{ACKNOWLEDGEMENTS}

We would like to thank Dr. Dolte and Dr. Bergfeld for permission to use the Women's AGA Quality of Life Questionnaire tool.

Funding Sources: Scientific Research Fund, Australian College of Dermatologists

The authors have no potential conflict of or competing interests to disclose.

\section{ABBREVIATIONS}

$$
\begin{array}{ll}
\text { AGA } & =\text { Androgenetic alopecia } \\
\text { FPHL } & =\text { Female pattern hair loss } \\
\text { QOL } & \text { Quality of life } \\
\text { FAA-QOL }= & \begin{array}{l}
\text { Female androgenetic alopecia quality of } \\
\text { life index }
\end{array}
\end{array}
$$

\section{REFERENCES}

[1] Cash TF. The psychology of hair loss and its implications for patient care. Clin Dermatol 2001; 19: 161-6.

[2] Sinclair RD. Fortnightly review: male pattern androgenetic alopecia. Br Med J 1998; 317: 865-9.

[3] Sinclair R, Jolley D, Mallari R, Magee J. The reliability of horizontally sectioned scalp biopsies in the diagnosis of chronic diffuse telogen hair loss in women. J Am Acad Dermatol 2004; 51(2): 189-99.

[4] Norwood O'TT. Male pattern baldness. Classification and incidence. South Med J 1975; 68: 1359-70.

[5] Olsen EA. Female pattern hair loss. J Amer Acad Dermatol 2001; 45: $\mathrm{S} 70-80$

[6] Ludwig E. Classification of the types of androgenic alopecia (common baldness) arising in the female sex. Br J Dermatol 1977; 97: 249-56.

[7] Gan, DCC, Sinclair RD. Prevalence of male and female pattern hair loss in Maryborough. J Invest Dermatol Symp Proc 2005; 10: 1849 .
[8] Budd D, Himmelberger D, Rhodes T, Cash TF, Girman CJ. The effects of hair loss in European men; A survey in four countries. Eur J Dermatol 2000; 10: 122-7.

[9] de Koning EBG, Passchier J, Dekker FW. Psychological problems with hair loss in general practice and the treatment policies of general practitioners. Psychol Rep 1990; 67: 775-8.

[10] Cash TF. Losing hair, losing points-the effects of male baldness on social impression formation. J Appl Soc Psychol 1990; 20: 154-67.

[11] Cash TF. The psychological effects of androgenetic alopecia in men. J Am Acad Dermatol 1992; 26: 926-31.

[12] Franzoi SL, Anderson J, Frommelt S. Individual differences in men's perceptions of and reactions to thinning hair. J Soc Psychol 1990; 130: 209-18.

[13] Girman CJ, Rhodes T, Lilly FRW, et al. Effects of self-perceived hair loss in a community sample of men. Dermatology 1998; 197: 223-9.

[14] Gosselin C. Hair loss, personality and attitudes. Pers Indiv Dif 1984; 5: 365-9.

[15] Roll S, Verinis JS. Stereotypes of scalp and facial hair as measured by the semantic differential. Psychol Rep 1971; 28: 975-80.

[16] van der Donk J, Passchier J, Dutree-Meulenberg ROGM, Stolz E, Verhage F. Psychologic characteristics of men with alopecia androgenetic and their modification. Int J Dermatol 1991; 30: 22-8.

[17] Wells PA, Wilmoth T, Russell RJH. Does fortune favour the bald? Psychological correlates of hair loss in males. Br J Psychol 1995; 86: 337-44.

[18] van der Donk J, Passchier J, Knegt-Junk KJ, et al. Psychological characteristics of women with androgenetic alopecia: a controlled study. Br J Dermatol 1991; 125: 248-52.

[19] Cash TF, Price VH, Savin RC. Psychological effects of androgenetic alopecia on women: Comparisons with balding men with female control subjects. J Am Acad Dermatol 1993; 29: 56875.

[20] van der Donk J, Hunfeld JAM, Passchier J, Knegt-Junk KJ, Nieboer C. Quality of life and maladjustment associated with hair loss in women with androgenetic alopecia. Soc Sci Med 1994; 38: 159-63.

[21] Sinclair R, Wewerinke M, Jolley D. Treatment of female pattern hair loss with oral antiandrogens. Br J Dermatol 2005; 152: 466-73.

[22] Sinclair RD, Dawber RPR. Androgenetic alopecia in men and women. Clin Dermatol 2001; 19: 167-78.

[23] Drake LA, Dinehart SM, Farmer ER, et al. Guidelines of care for androgenetic alopecia. J Am Acad Dermatol 1996; 35; 465-8.

[24] Guyatt GH, Feeny DH, Patrick DL. Measuring health-related quality of life. Ann In Med 1993; 118: 622-9.

[25] Patrick DL, Deyo RA. Generic and disease-specific measures in assessing health status and quality of life. Med Care 1989; 27: S217-31.

[26] Dolte KS, Girman CJ, Hartmaier S, Bergfeld W, Waldstreicher J. Development of a health-related quality of life questionnaire for women with androgenetic alopecia. Clin Exp Dermatol 2000; 25: $637-42$.

[27] Schmidt S, Fischer TW, Chren MM, Strauss BM, Elsner P. Strategies of coping and quality of life in women with alopecia. $\mathrm{Br}$ J Dermatol 2001; 144: 1038-43.

[28] Girman CJ, Hartmaier S, Roberts J, Bergfeld W, Waldstreicher J. Patient-perceived importance of negative effects of androgenetic alopecia in women. J Womens Health Gend Based Med 1999; 8: $1091-5$.

[29] Cartwright T, Endean N, Porter A. Illness perceptions, coping and quality of life in patients with alopecia. Br J Dermatol 2009; 160: $1034-9$. 